\title{
Scaling the Microrheology of Living Cells
}

\author{
Ben Fabry, ${ }^{1, *}$ Geoffrey N. Maksym, ${ }^{2}$ James P. Butler, ${ }^{1}$ Michael Glogauer, ${ }^{3}$ Daniel Navajas, ${ }^{4}$ and Jeffrey J. Fredberg ${ }^{1}$ \\ ${ }^{1}$ Physiology Program, Harvard School of Public Health, 665 Huntington Avenue, Boston, Massachusetts 02115 \\ ${ }^{2}$ School of Biomedical Engineering, Dalhousie University, 5981 University Avenue, Halifax B3H 3J5, Canada \\ ${ }^{3}$ Division of Experimental Medicine, Harvard Medical School, 221 Longwood Avenue, Boston, Massachusetts 02115 \\ ${ }^{4}$ Unitat Biofísica i Bioenginyeria, Universitat de Barcelona-IDIBAPS, Casanova 143, 08036 Barcelona, Spain
}

(Received 27 November 2000; published 13 September 2001)

\begin{abstract}
We report a scaling law that governs both the elastic and frictional properties of a wide variety of living cell types, over a wide range of time scales and under a variety of biological interventions. This scaling identifies these cells as soft glassy materials existing close to a glass transition, and implies that cytoskeletal proteins may regulate cell mechanical properties mainly by modulating the effective noise temperature of the matrix. The practical implications are that the effective noise temperature is an easily quantified measure of the ability of the cytoskeleton to deform, flow, and reorganize.
\end{abstract}

DOI: $10.1103 /$ PhysRevLett.87.148102

Mechanical stresses and resulting deformations play central roles in cell contraction, spreading, crawling, invasion, wound healing, and division, and have been implicated in regulation of protein and DNA synthesis and programed cell death [1]. If the cytoskeleton were simply an elastic body, it would maintain its structural integrity by developing internal elastic stresses to counterbalance whatever force fields it might be subject to. However, those same elastic stresses would tend to oppose - or even preclude altogether-other essential mechanical functions such as cell spreading, crawling, extravasation, invasion, division, and contraction, all of which require the cell to "flow" similar to a liquid. A liquidlike cell, however, would be unable to maintain its structural integrity. The classical resolution of this paradox has been the idea that cytoskeletal polymers go through a sol-gel transition, allowing the cytoskeleton to be fluidlike in some circumstances (the sol phase) and solidlike in others (the gel phase) [2-4]. The data presented here suggest that, rather than being thought of as a gel, the cytoskeleton may be thought of more properly as a glassy material existing close to a glass transition, and that disorder and metastability may be essential features underlying its mechanical functions.

We coated ferrimagnetic microbeads ( $4.5 \mu \mathrm{m}$ diameter) with a synthetic RGD (Arg-Gly-Asp)-containing peptide (Integra Life Sciences) and bound them specifically to integrin receptors on the surface of human airway smooth muscle (HASM) cells (Figs. 1a and 1b). The beads were magnetized horizontally and then twisted vertically by an external homogeneous magnetic field that was varying sinusoidally in time (Fig. 1c).

Lateral bead displacement in response to the resulting oscillatory torque was detected by a charge-coupled device camera (JAI CV-M10) mounted on an inverted microscope. Image acquisition (exposure time: $0.1 \mathrm{~ms}$ ) was phase locked to the twisting field so that 16 images were acquired during each twisting cycle. Heterodyning was used at twisting frequencies $>1 \mathrm{~Hz}$. The images were analyzed using an intensity-weighted center-of-mass algo-
PACS numbers: $87.16 . \mathrm{Ka}$, 64.70.Pf, 83.85.Vb, 87.19.Rr

rithm in which subpixel arithmetic allowed the determination of bead position with an accuracy of $5 \mathrm{~nm}(\mathrm{rms})$.

The specific torque, $T$, is the mechanical torque per bead volume, and has dimensions of stress $(\mathrm{Pa})$. The ratio of the complex specific torque $\tilde{T}$ to the resulting complex bead displacement $\tilde{d}$ defines a complex elastic modulus of the cell $\tilde{g}=\tilde{T}(f) / \tilde{d}(f)$, and has dimensions of $\mathrm{Pa} / \mathrm{nm}$. For each bead, we computed the elastic modulus $g^{\prime}$ (the real part of $\tilde{g}$ ), the loss modulus $g^{\prime \prime}$ (the imaginary part of $\tilde{g}$ ), and the loss tangent $\eta$ (the ratio $g^{\prime \prime} / g^{\prime}$ ). These measurements could be transformed into traditional elastic and loss moduli by a geometric factor $\alpha$ that depends on the shape and thickness of the cell, and the degree of bead embedding, where $\tilde{G}=\alpha \tilde{g}$. Finite element analysis of cell deformation for a representative bead-cell geometry (assuming homogeneous and isotropic elastic properties with $10 \%$ of the bead diameter embedded in a cell $5 \mu \mathrm{m}$ high) sets $\alpha$ to $6.8 \mu \mathrm{m}$. This geometric factor need serve only as a rough approximation because it cancels out in the scaling procedure described below, which is model independent.
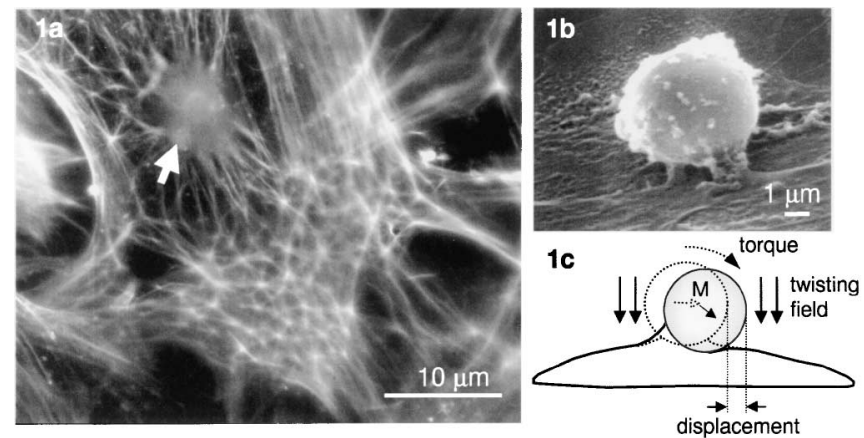

FIG. 1. (a) Ferrimagnetic beads (arrow) bind avidly to the actin cytoskeleton (stained with phalloidin) of HASM cells via cell adhesion molecules (integrins). (b) Scanning electron microscopy of a bead bound to the cell surface. (c) A magnetic field introduces a torque which causes the bead to rotate and to displace. $M$ denotes the direction of the bead's magnetic moment. 
Bead displacement amplitude followed a log-normal distribution that has been analyzed in detail elsewhere [5]. The loss tangent and the frequency dependence of $G^{\prime}$ and $G^{\prime \prime}$ varied little between beads, however. Importantly, $G^{\prime}$ and $G^{\prime \prime}$ were found to be constant over a wide range of specific torque amplitudes $(1.8-130 \mathrm{~Pa})$ and lateral bead displacement amplitudes (5-500 nm), implying linear mechanical behavior in this range.

$G^{\prime}$ increased weakly with frequency and followed a power law, $f^{0.17}$ (Fig. 2a). $G^{\prime \prime}$ also followed a weak power law at low frequencies, but beyond $10 \mathrm{~Hz}$ progressively stronger frequency dependence emerged, approaching but never quite attaining a power-law exponent of 1 , which would be characteristic of a Newtonian viscosity (Fig. 2b). $G^{\prime}$ was larger than $G^{\prime \prime}$ for twisting frequencies below $300 \mathrm{~Hz}$ (Fig. 2d), and the loss tangent remained almost constant between 0.3 and 0.4 over the lower four frequency decades. We found similar behavior when human bronchial epithelial cells were probed between 0.1 and $10 \mathrm{~Hz}$ using atomic force microscopy (data not shown). Comparable findings over more limited ranges of

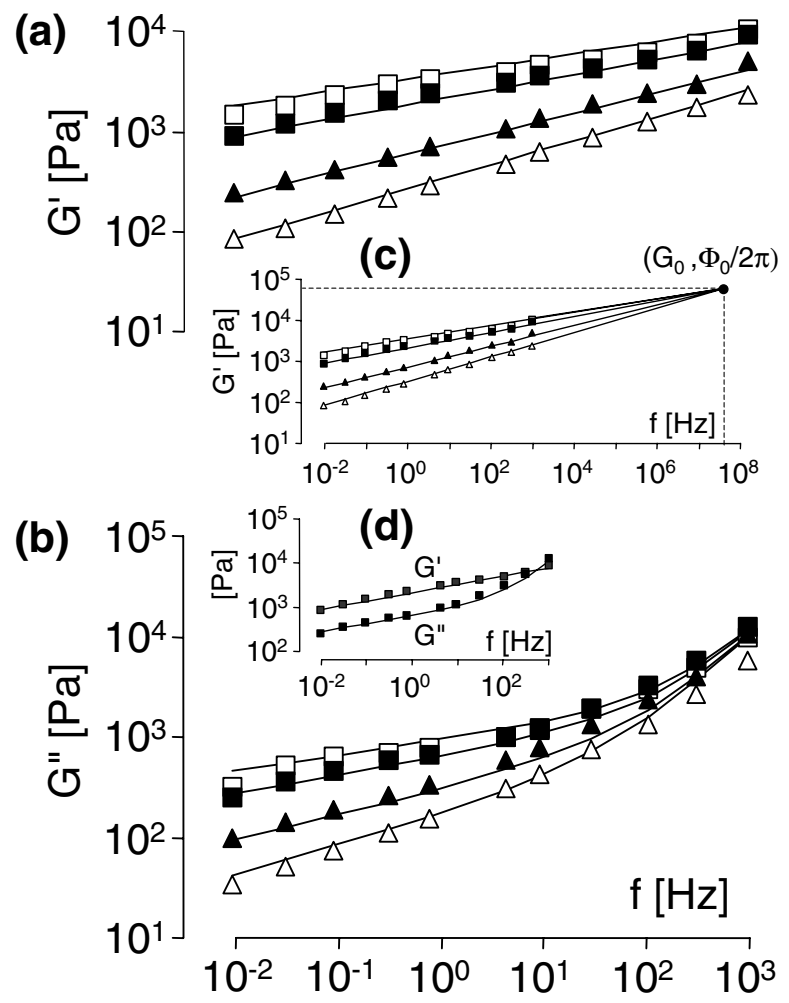

FIG. 2. $G^{\prime}$ (a), $G^{\prime \prime}$ (b) of HASM cells vs frequency under control conditions $(\boldsymbol{\square}, n=256)$, and after 10 min treatment with histamine $\left[10^{-4} \mathrm{M}\right](\square, n=195)$, DBcAMP $\left[10^{-3} \mathrm{M}\right]$ $(\boldsymbol{\Delta}, n=239)$ and cytochalasin $D\left[2 \times 10^{-6} \mathrm{M}\right](\triangle, n=171)$. The solid lines are the fit of Eq. (1) to the data and have been obtained by minimizing mean square residuals for all $G^{\prime}$ and $G^{\prime \prime}$ data over the measurement bandwidth $\left(G_{0}=53.6 \mathrm{kPa}\right.$, $\left.\Phi_{0}=2.5 \times 10^{8} \mathrm{rad} / \mathrm{s}, \mu=1.41 \mathrm{Pas}\right)$. $G$ was computed as $\alpha \tilde{g}$, with $\alpha$ being derived from a finite element analysis of a representative bead-cell geometry. (c) Extrapolation of Eq. (1) to higher frequencies yields crossover at coordinate $\left(G_{0}, \Phi_{0}\right)$. (d) $G^{\prime}$ and $G^{\prime \prime}$ vs $f$ under control conditions. frequency have been obtained with different techniques in several other cell types [6-10]. Experimental models of cell rheology using semidilute F-actin solutions [11], however, display rheological characteristics that can differ rather substantially from the weak power law behavior observed in living cells (Fig. 2).

Addition of the contractile agonist histamine to HASM cells caused $G^{\prime}$ and $G^{\prime \prime}$ to increase (Figs. 2a and 2b). The loss tangent and the exponent of the power law fell slightly. By contrast, ablating baseline contractile tone with $\mathrm{N}^{6}, 2^{\prime}$-O-dibutyryladenosine $3^{\prime}, 5^{\prime}$-cyclic monophosphate (DBcAMP) caused $G^{\prime}$ and $G^{\prime \prime}$ to fall, and the loss tangent and exponent to increase. When actin filaments were disrupted with cytochalasin $D, G^{\prime}$ and $G^{\prime \prime}$ fell even more, and the loss tangent and exponent increased further.

These data, taken together, cannot be explained by receptor-ligand dynamics as would be the case if there were repetitive peeling of the bead away from the cell surface and subsequent reattachment with each bead oscillation. Neither can these data be readily explained by changes in cell membrane mechanics. Rather, these data are more consistent with the notion that the bead binds avidly to the cytoskeleton via focal adhesions, and that the cytoskeleton is deformed as the bead rotates (Fig. 1). Accordingly, $G^{\prime}$ and $G^{\prime \prime}$ predominantly reflect the mechanical properties of the cytoskeleton. This view is further supported by experiments using beads coated with acetylated low density lipoprotein (acLDL); those beads bind to scavenger receptors which do not form focal adhesion complexes [12]. We found that baseline values of $G^{\prime}$ and $G^{\prime \prime}$ measured with acLDL-coated beads were smaller by fivefold compared with beads bound to integrins, and demonstrated an attenuated response to contractile agonists.

Structural damping.-The data in Fig. 2 conform closely to an empirical law that is known in the engineering literature as structural damping or hysteretic damping [13-18]. Accordingly,

$$
\begin{aligned}
\tilde{G}= & G_{0}\left(\frac{\omega}{\Phi_{0}}\right)^{x-1}(1+j \bar{\eta}) \Gamma(2-x) \cos \frac{\pi}{2}(x-1) \\
& +j \omega \mu,
\end{aligned}
$$

where $\bar{\eta}=\tan (x-1) \pi / 2$, and $\omega$ is the radian frequency $2 \pi f . G_{0}$ and $\Phi_{0}$ are scale factors for stiffness and frequency, respectively, $\Gamma$ denotes the gamma function, $\mu$ is a Newtonian viscous term, and $j$ is the unit imaginary number $\sqrt{-1}$. $G_{0}$ and $\mu$ depend on bead-cell geometry. $\bar{\eta}$ has been called the hysteresivity or the structural damping coefficient. The elastic modulus corresponds to the real part of Eq. (1), which increases for all $\omega$ according to the power-law exponent, $x-1$. The loss modulus corresponds to the imaginary part of Eq. (1) and includes a component that is a frequency-independent fraction $(\bar{\eta})$ of the elastic modulus; such a direct coupling of the loss modulus to the elastic modulus is the characteristic feature of structural damping behavior [18]. The loss modulus also includes a Newtonian viscous term, $j \omega \mu$, which comes into play only at higher frequencies. At low frequencies, 
the loss tangent $\eta$ approximates $\bar{\eta}$. In the limit that $x$ approaches unity, the power-law slope approaches zero, $G^{\prime}$ approaches $G_{0}$, and $\bar{\eta}$ approaches zero. Thus, Eq. (1) describes a relationship between changes of the exponent of the power law and the transition from solidlike $(x=1)$ to fluidlike $(x=2)$ behavior.

Unexpectedly, we could fit Eq. (1) to the data in Fig. 2 using only a single value for $G_{0}$ and for $\Phi_{0}$. This implies a common intersection of the $G^{\prime}$ vs $f$ curves when extrapolated outside the measurement range (Fig. 2c). Further, $\mu$ was found to vary little with cellular manipulations. This leaves $x$ as the sole free parameter in Eq. (1).

Master curves. - We estimated $x$ directly from the measured power-law dependence of $G^{\prime}$ on $f$. We then defined a normalized stiffness $\mathbf{G}$ as being $G^{\prime}$ measured at $0.75 \mathrm{~Hz}$ (an arbitrary choice) divided by $G_{0}$, which causes the geometric factor $\alpha$ to cancel out. G vs $x$ for all manipulations followed the relationship shown in Fig. 3a (black symbols). Similarly, $\eta$ (measured as the ratio of $G^{\prime \prime} / G^{\prime}$ at $0.75 \mathrm{~Hz}$ ) vs $x$ followed the relationship shown in Fig. 3b (black symbols). When we studied a variety of cell types, corresponding data were found in every case to conform to Eq. (1); $G_{0}$ and $\mu$ differed between cell types, but $\Phi_{0}$ was invariant $\left(2.5 \times 10^{8} \mathrm{rad} / \mathrm{s}\right)$.

Despite important differences in the elastic and frictional material properties, all data collapsed onto the very same relationships as did the HASM cells (Fig. 3). These relationships thus represent universal master curves in that a single free parameter, $x$, defined the constitutive elastic and frictional behaviors for a variety of cytoskeletal manipulations, for five frequency decades, and for diverse cell types. The normalization that leads to these master curves is model independent. Nonetheless, these master curves fell close to the predictions from Eq. (1) (solid black lines).

Soft glassy materials. - An attractive physical interpretation for the parameter $x$ comes from the physics of soft glassy materials (SGM). The empirical criteria that define this class of material are that $G^{\prime}$ and $G^{\prime \prime}$ increase with
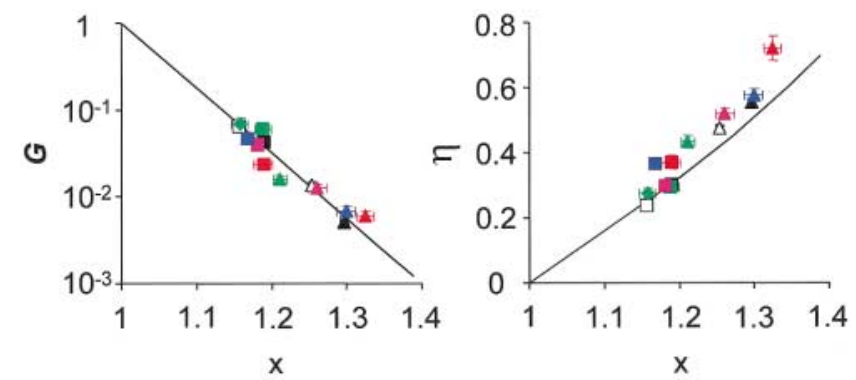

FIG. 3 (color). Master curves ( $\mathbf{G}$ and $\eta$ at $0.75 \mathrm{~Hz}$ vs $x$ ) of HASM cells (black, $n=256$ ), human bronchial epithelial cells (blue, $n=142$ ), mouse embryonic carcinoma cells (F9) cells (pink, $n=50$ ), mouse macrophages (J774A.1) (red, $n=46)$ and human neutrophils (green, $n=42$ ) under control conditions $(\boldsymbol{\square})$, treatment with histamine $(\square)$, FMLP $(\diamond)$, DBcAMP $(\triangle)$, and cytoD $(\boldsymbol{\Delta})$. Equation (1) predicts the black solid curves: $\ln \mathbf{G}=(x-1) \ln \left(\omega / \Phi_{0}\right)$ with $\Phi_{0}=2.5 \times 10^{8} \mathrm{rad} / \mathrm{s}$, and $\bar{\eta}=\tan [(x-1) \pi / 2]$. Error bars indicate \pm 1 standard error. weak power-law dependencies upon frequency, and that the loss tangent is frequency insensitive and of the order $0.1[19,20]$. Accordingly, the data in Figs. 2 and 3 establish that the cells studied here behaved as soft glassy materials. Surprisingly, other materials in the class include foams, emulsions, colloid suspensions, pastes, and slurries $[20,21]$. Existing models of viscoelasticity have thus far failed to provide a satisfactory explanation of the rheology of SGMs $[17,20]$. Moreover, it is unclear how such diverse systems express mechanical behavior that is so much alike.

It has been suggested by Sollich [20] that the common rheological features of this class may be not so much a reflection of the particular molecules or molecular mechanisms as they are a reflection of a generic system property at some higher level of structural organization [20,22]. The generic properties that these SGMs share are that each is composed of elements that are discrete, numerous, aggregated with one another via weak attractive interactions, and arrayed in a geometry that is structurally disordered and metastable. Glassy behavior in such systems is a consequence of structural relaxation in which the elements have to cross energy barriers that are large compared with thermal energies. A disordered metastable configuration arises if the elements are quenched before they can attain a minimum energy (equilibrium) state.

Sollich [20] extended the earlier work of Bouchaud [23] to develop a unified theory of soft glassy rheology (SGR). SGR theory considers that the individual elements of the matrix exist within an energy landscape containing many wells, or traps, formed by neighboring elements. These traps are of differing energy depth, and each is regarded as being so deep that the element is unable to escape the well by thermal fluctuations. Instead, elements are imagined to be agitated and rearranged by their mutual interactions within the matrix [20]. A clear notion of the source of the agitation remains to be identified, but this agitation can be represented nonetheless by an effective temperature, or noise level, $x$. The elements are unable to escape their wells when $x=1$, in which case the system is perfectly elastic. When $x>1$, however, the elements can hop randomly between wells and, as a result, the system can flow and become disordered. Flow and disorder are the essential features of a glassy material. Thus, as $x$ approaches unity from above, the system approaches a glass transition. In the limit of linear responses at low frequencies, SGR theory [20] leads to a constitutive equation that we now identify as being identical to the structural damping law [Eq. (1)].

Accordingly, SGR theory provides the following interpretation of the free parameter $x$ and the master curves reported here (Fig. 3). Agents that activate the contractile apparatus and/or polymerize cytoskeletal proteins move the cell towards a glass transition. A decreasing noise temperature is consistent with the formation of structure, which in turn may be associated with the notion of de Gennes [24] that chemical bonding is equivalent to attractive interactions; the system becomes more ordered and approaches a frozen state. Conversely, relaxing 
agonists and agents that disrupt the cytoskeleton cause the system to become more disordered and move towards a fluid state. SGR theory also identifies the parameter $G_{0}$ in Eq. (1) as being the stiffness at the glass transition, and the parameter $\Phi_{0}$ as being the frequency at which elements attempt to escape their energy wells. If so, then $G_{0}$ represents an upper limit of stiffness and might correspond to the stiffness defined by the purely elastic cytoskeletal model of Satcher and Dewey [25].

Time-scale invariance.-The attempt rate $\Phi_{0}$ is not to be confused with the frequency at which elements escape their energy wells, and does not correspond to a relaxation time scale [19]. Rather, the inverse Fourier transform of Eq. (1) predicts a wide range of relaxation time scales $[13,15]$, relaxing according to the power law $t^{1-x}$. Power-law behavior implies that the relaxation processes are not tied to any particular internal time scale.

Regulation of cell mechanics.-Although glasslike behavior has been reported for protein macromolecules [26], we do not necessarily expect that SGR theory will apply to mechanical properties at the level of discrete molecular interactions. Moreover, the data presented here were restricted to small amplitude deformations coupled to the cytoskeleton via integrins, and to a limited subset of cells and substrates. Nonetheless, these data lead to the prospect of a major conceptual simplification. The observation of universal master relationships describing cell rheology supports the hypothesis that details of the molecular interactions are manifested largely through their effects on $x$. This behavior is reminiscent of statistical mechanics in systems that have only a weak dependence of aggregate behavior on the details of the molecular interactions. We speculate that some key functions of diverse cytoskeletal proteins, such as their influence on the ability of the cytoskeleton to deform, flow, and reorganize, may be understood mainly through their ability to modulate $x$. We have no evidence, however, that $x$ is a regulated variable. SGR theory cannot predict how much a given drug might change $x$, but it does predict the effects of any druginduced change in $x$ on cytoskeletal rheology. As a practical matter, it provides a conceptual framework for assays of drug potency.

The rheological behavior reported here stands in contrast with prevailing theories of cell mechanics, which hold that cell rheology arises from an interaction of distinct elastic and viscous components expressing a limited range of characteristic relaxation times. Except at frequencies in excess of several tens of $\mathrm{Hz}$, the data reported here support the hypothesis that the locus of both the frictional and the elastic stresses is within formed structures of the cytoskeleton $[9,17,20]$, and that the dominant frictional stress does not correspond to a viscous stress.

To the extent that the concept of the sol-gel transition can be applied usefully to the mechanics of these formed cytoskeletal structures, the data presented here suggest that the gel phase may be thought of more properly as a glassy material existing close to a glass transition; if so, then disorder and metastability may be essential features underlying mechanical functions of the cytoskeleton. A key thermodynamic distinction between the sol-gel transition (in a strong gel) and the glass transition is that the former is an equilibrium process, with structural elements found at energy minima, whereas the latter is a nonequilibrium process with elements trapped in metastable states. Finally, we wish to point out that it is unclear at this time how the level of metastability and intracellular agitation as expressed by the effective noise temperature might depend upon how far the cell departs from thermodynamic equilibrium due to its energy metabolism, but this would seem to be an important question.

We thank S. Shore, P. Moore, W. Goldmann, C.F. Dewey, D. Tschumperlin, S. Mijailovich, W. Möller, M. Harp, R. Panettieri, R. Rogers, and D. Weitz. This study was supported by NIH HL33009, HL65960, HL59682, and DGESIC-PM980027.

*Corresponding author.

Email address: bfabry@hsph.harvard.edu

[1] P. A. Janmey, Physiol. Rev. 78, 763 (1998).

[2] P. A. Janmey et al., Nature (London) 345, 89 (1990).

[3] T. P. Stossel, Science 260, 1086 (1993).

[4] M. Tempel, G. Isenberg, and E. Sackmann, Phys. Rev. E 54, 1802 (1996).

[5] B. Fabry et al., J. Appl. Physiol. 91, 986 (2001).

[6] S. G. Shroff, D. R. Saner, and R. Lal, Am. J. Physiol. 269, C286 (1995).

[7] R. E. Mahaffy et al., Phys. Rev. Lett. 85, 880 (2000).

[8] W. H. Goldmann and R. M. Ezzell, Exp. Cell Res. 226, 234 (1996).

[9] G. N. Maksym et al., J. Appl. Physiol. 89, 1619 (2000).

[10] S. Yamada, D. Wirtz, and S. C. Kuo, Biophys. J. 78, 1736 (2000).

[11] T. Gisler and D. A. Weitz, Phys. Rev. Lett. 82, 1606 (1999).

[12] N. Wang, J. P. Butler, and D. E. Ingber, Science 260, 1124 (1993).

[13] W. Weber, Ann. Phys. Chem. 54, 1 (1841).

[14] F. Kohlrausch, Ann. Phys. Chem. 128, 1 (1866).

[15] J. Hildebrandt, Bull. Math. Biophys. 31, 651 (1969).

[16] Y. C. Fung, Am. J. Physiol. 213, 1532 (1967).

[17] J. J. Fredberg and D. Stamenovic, J. Appl. Physiol. 67, 2408 (1989).

[18] S. H. Crandall, J. Sound Vib. 11, 3 (1970).

[19] P. Sollich et al., Phys. Rev. Lett. 78, 2020 (1997).

[20] P. Sollich, Phys. Rev. E 58, 738 (1998).

[21] V. Trappe and D. Weitz, Phys. Rev. Lett. 85, 449 (2000).

[22] J. Phillips, Rep. Prog. Phys. 59, 1133 (1996).

[23] J. Bouchaud, J. Phys. I (France) 2, 1705 (1992).

[24] P.G. de Gennes, Scaling Concepts in Polymer Physics (Cornell University Press, Ithaca, 1979), pp. 134-152.

[25] R. L. Satcher, Jr. and C. F. Dewey, Jr., Biophys. J. 71, 109 (1996).

[26] A. L. Lee and A. J. Wand, Nature (London) 411, 501 (2001). 